\title{
Economía y sociología de la educación: los retos del siglo XXI
}

Para afrontar con éxito los retos que en materia educativa impone el siglo XXI es necesario introducir transformaciones decisivas en los sistemas escolares y en el proceso de enseñanza-aprendizaje. La educación de hoy implica nuevas exigencias en todos los niveles del sistema, desde el preescolar hasta la educación superior; así lo reconoce y analiza el copioso acervo de literatura teórica y los estudios aplicados que desde el mundo desarrollado hasta los países emergentes se ha producido en las últimas dos décadas.

La economía y la sociología de la educación no han estado ajenas a tales preocupaciones y por ello han experimentado en las últimas dos décadas importantes progresos. El campo de estudio de la economía de la educación hoy día trasciende las preocupaciones intelectuales de los trabajos pioneros de Shultz y Becker e incluso ha logrado ampliar el campo de análisis más allá de las relaciones entre educación, salarios y crecimiento económico. Han surgido nuevas preguntas de investigación, en muchos de los casos relacionadas con los potenciales de logro educativo en entornos de elevada desigualdad social; también han surgido inquietudes de gran relevancia en lo que concierne al estudio de la calidad educativa, las estrategias para su mejoramiento y el impulso de políticas de intervención orientadas a promover la igualdad de oportunidades en el acceso y calidad de sus beneficios.

Este número pretende precisamente enriquecer la discusión que se ha derivado alrededor de la evaluación de la calidad educativa y sus implicaciones, a la vez que busca ampliar el debate que generan las nuevas perspectivas desde la sociología y la economía de la educación, en los que aparecen interrogantes esenciales como ¿Cuáles son los factores asociados al desempeño escolar? ¿Cuál es el rol de los entornos socioeconómicos y sociodemográficos a la hora de explicar las diferencias en los logros educativos? ¿Persiste el trade off entre eficiencia y calidad educativa? ¿Cómo inciden las prácticas pedagógicas en los resultados de las pruebas estandarizadas? ¿Es la educación de calidad un bien de club que profundiza las desigualdades sociales? ¿Es la educación un bien público o un servicio público que solo lo puede prestar el Estado? ¿Es un derecho de todos los miembros de la sociedad?

Estos y otros interrogantes orientados a entender el impacto que los cambios sociales y económicos de los últimos años han tenido sobre la educación y la institución escolar, así como los retos que estas transformaciones imponen al siglo XXI, configuran el tema central de esta edición de la Revista Sociedad y Economía. 
El primer artículo aborda la discusión sobre desigualdad educativa y sus implicaciones en las trayectorias de acumulación del capital humano bajo diferentes regímenes de financiación. Realiza algunos ejercicios de simulación dinámica y sugiere nuevas líneas de investigación para el diseño de políticas gubernamentales.

El trabajo de Correa Fonnegra estudia los factores asociados al desempeño académico de los estudiantes y la incidencia de los diferenciales de género en Colombia. Apoya su investigación en los microdatos de las pruebas TIMSS 2007 en matemáticas y utiliza modelos lineales jerárquicos para la contrastación de sus hipótesis.

El artículo sobre clasificación de colegios de Carlos Alberto Gómez utiliza modelos marginales aplicados a las pruebas Saber 11 en Colombia. Establece diferentes categorías de desempeño de las instituciones y su ordenamiento.

El estudio sobre rendimiento escolar en matemáticas para tercer grado de educación básica primaria en América Latina de Zambrano Jurado utiliza los datos de las pruebas Serce 2006 que incluye varios países de la región y aplica estructuras lineales jerárquicas en tres niveles para evaluar la incidencia de variables como el género, tipo de escuela y repitencia, entre otras.

El trabajo de Beltrán y Berrío se centra en del desempeño laboral de los egresados del Departamento de Sociología de la Universidad Nacional de Colombia. El estudio concluye que existe un desfase entre las expectativas de los estudiantes y las posibilidades reales de inserción a los mercados parciales de trabajo (investigación, docencia, consultoría y trabajo aplicado en comunidades).

La investigación de Castro Heredia y Raffo López introduce algunas consideraciones sobre la estructura curricular de las carreras de economía en el país. Los autores encuentran que efectivamente existe convergencia en sus estructuras que sugiere la adaptación de un modelo líder-seguidor que puede ir en detrimento del sello diferenciador que cada región podría instaurar en sus programas.

El trabajo de Salazar y Ordóñez evalúa las experiencias de Estados Unidos, Finlandia, Corea del Sur, Brasil y Chile en la introducción de reformas. Los autores encuentran que la flexibilidad, la participación, la cohesión social y la adopción de sistemas adaptativos complejos constituyen factores claves a la hora de explicar los casos exitosos.

Tal y como se puede ver en los artículos que conforman el tema central, se trata de un campo fructífero, en plena ebullición y con promisorias perspectivas de desarrollo en los próximos años. Estos trabajos han permitido ver que a pesar de que los sistemas escolares han trasegado desde dimensiones simples a estructuras de enorme complejidad en los ámbitos nacionales y locales, no se ha logrado responder aún con eficacia a las pretensiones cada vez más exigentes de calidad.

No se trata, sin embargo, de introducir reformas y contrarreformas cargadas de supuestas innovaciones y políticas ad hoc que no tienen en cuenta la integralidad del sistema y que abordan de manera aislada cada uno de sus componentes; se trata más bien de abordar con inteligencia el diseño 
de las escuelas, colegios y universidades del mañana, su transformación en instituciones eficaces, de calidad y que brinden mayores y mejores oportunidades de inserción en las dinámicas sociales que impone el siglo XXI.

Todos estos estudios, por supuesto, aportan a la construcción de capacidades para adelantar investigación de calidad y fortalecer el vínculo entre investigación, política pública y las prácticas educativas que nos permitan pensar en las escuelas y universidades del mañana

Harvy Vivas Pacheco

Profesor Facultad de Ciencias Sociales y Económicas

(c) (i) Este trabajo está bajo la licencia Creative Commons Attribution 3.0

¿Cómo citar este artículo? / How to quote this article?

Vivas, Harvy «Presentación tema central edición $\mathrm{N}^{\circ}$ 30. Economía y sociología de la educación: los retos del siglo XXI». Sociedad y economía, No. 30 (enero - junio 2016): 11-13. 